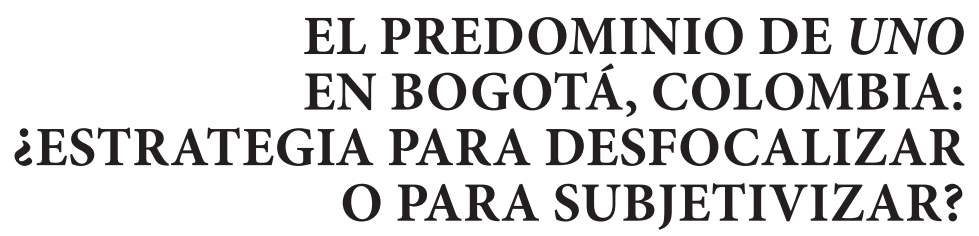

EL PREDOMINIO DE UNO EN BOGOTA, COLOMBIA: O PARA SUBJETIVIZAR? 
Este artículo analiza los factores semántico-pragmáticos que influyen en el uso del pronombre uno en el habla de Bogotá. Mediante un enfoque variacionista y con base en los resultados del programa estadístico Goldvarb, se advierte una reducción de la característica impersonal de uno, pues se utiliza principalmente como marcador de la postura del hablante (subjetivización del discurso) más que como mecanismo para disminuir su protagonismo. Los resultados de las variables de clase semántica del verbo, tipo de discurso y clase de entrevista aportan evidencia acerca de la utilización de uno casi con las mismas funciones referenciales de yo con verbos emotivos, mentales y estativos, en narraciones y situaciones hipotéticas, y en interacciones informales y espontáneas. Estos resultados apuntan a la pérdida de la antigua connotación socio-pragmática de un sobreuso característico de los individuos con menor nivel económico y educativo.

Palabras clave: español de Colombia, desfocalización, impersonalización, pronombres, subjetivización

This article analyzes the semantic and pragmatic factors influencing the use of the impersonal uno in the Spanish of Bogota. By means of a variationist approach, and based on the results from the statistical program Goldvarb, we note a reduction of the degree of impersonality expressed by uno, because it is used more as a marker of speaker stance (subjectification of discourse), than a mechanism to reduce prominence. The results of variables of verbal semantics, type of discourse, and kind of interview support that uno is used almost with the same referential functions as $y o$ with verbs of emotion, mental activities and states, narrations and hypothetical situations, and in informal and spontaneous interactions. These results suggest that it has lost its former socio-pragmatic connotation as an overuse typical of individuals from lower economic and education levels.

KeY wORDS: Colombian Spanish, defocusing, impersonalization, pronouns, subjectification

RECEPCIÓN: 29/05/2015

ACEPTACIÓN: 25/07/2015 


\title{
EL PREDOMINIO DE UNO EN BOGOTÁ, COLOMBIA: ¿ESTRATEGIA PARA DESFOCALIZAR O PARA SUBJETIVIZAR?
}

\author{
Luz Marcela Hurtado \\ Central Michigan University
}

\section{Introducción}

Desde una perspectiva comunicativa-funcional, las construcciones impersonales han sido consideradas como mecanismos de desfocalización con los cuales se intenta "disminuir o silenciar la importancia del papel que juega la persona referida en el estado de cosas descrito" (Haverkate, 1987: 47). Para algunos autores, cada forma indica un grado específico de impersonalidad. Por ejemplo, Company y Pozas (2009) señalan que estrategias como la segunda persona singular, uno, los indefinidos compuestos (ej. cualquiera), se, la tercera persona plural, la pasiva perifrástica (ser + participio) y los verbos meteorológicos y temporales, entre otros, "pueden expresar la impersonalidad en grados diversos, desde una atenuación débil del sujeto hasta predicados en que no es posible asignar un sujeto ni gramatical ni lógico 
de la acción significada por el verbo" (1075-1076). Sin embargo, tras la comparación del pronombre indefinido uno, el se impersonal y los usos generalizadores de la segunda persona tú y $U d$., algunos autores consideran que estas formas pueden llegar a coincidir y cumplir con la triple función de (1) ocultar a un agente o reducir el protagonismo del hablante (Haverkate, 1987; Muñiz Cachón, 1998), (2) integrar al hablante, involucrar al interlocutor y aludir a un grupo y (3) denotar a la humanidad entera (Siewierska, 2008a: 116); es decir, son versátiles en cuanto a su interpretación referencial (Casielles, 1996: 376; Hernanz, 1990: 160).

Para otros autores como Fernández (2008: 223-227), a pesar de que formas como la segunda persona, uno y se pueden incluir al hablante, al oyente y a otra persona, cada una refleja una diferencia de perspectiva, o sea, la manera como el individuo decide presentar la información. Algunos estudios de corte empírico, basados en el análisis de muestras orales, también han mostrado usos impersonales particulares a ciertas variedades de español, condicionados por factores de tipo semántico-pragmático y social. Por ejemplo, el empleo de la segunda persona tú y de uno en la conversación oral informal en los madrileños (Fernández, 2008); la utilización de un se corporativo (que incluye al hablante y a su empresa) en las entrevistas de negocios del periódico español El País (Martínez-Linares, 2009); el uso de tú para presentar información personal y privativa, y de uno con referencia universal en el español de Chile (González Vergara y Hugo Rojas, 2012); el favorecimiento de tú impersonal y uno en la narración de experiencias personales de puertorriqueños bilingües, y el predominio de $s e$ impersonal en los 
monolingües (Morales, 1995); la alta frecuencia de uno en el habla urbana colombiana de Miami y Bogotá, proveniente de niveles de ocupación-educación bajo y medio (Hurtado, 2012), yen el habla de los adultos caraqueños (Guirado, 2011).

Por la variabilidad observada en el caso particular de uno, el presente estudio se ubica en una perspectiva semántico-pragmática combinada con un enfoque variacionista para indagar los posibles determinantes de su alta frecuencia en Bogotá. Se intenta explorar en qué circunstancias se utiliza, y por qué prevalece una forma considerada típica del habla popular. Es de interés especial el estudio de la variedad de Bogotá, ya que ha gozado de la reputación de ser una variedad de prestigio dentro de Colombia y el mundo hispánico (Montes Giraldo et al., 1998: 17), y se considera como modelo a seguir para los colombianos con más nivel de educación (Orozco y File-Muriel, 2012: 11). La predilección de una forma previamente característica de los estratos socioculturales bajos con un nivel educativo de primaria (Guantiva Acosta, 2000: 259), puede constituir un reflejo de las nuevas dinámicas de la ciudad, un cambio en la percepción de lo anteriormente considerado de prestigio. Por lo tanto, este trabajo intenta resolver los siguientes interrogantes: (1) ¿Por qué se está propagando a otros sectores sociales un uso frecuente de las clases populares? (2) ¿Se tratará de un cambio del significado impersonal de uno como encubridor de yo a uno casi equivalente a las funciones pronominales de yo? (3) ¿Constituye solamente un mecanismo mediante el cual el bogotano consigue distanciarse de sus enunciados o, en realidad, un acto inherentemente subjetivo y evaluativo con el que intenta realzar su posición? 
Ya que el hablante incorpora sus actitudes y creencias a través de mecanismos de subjetivización (Traugott, 2010: 35), el objetivo general consiste en indagar si se ha debilitado el significado referencial original de uno (estrategia para desfocalizar), y si se han expandido o convencionalizado sus funciones en la comunidad (enriquecimiento pragmático) con el fin de expresar las experiencias del hablante. Por eso, entre los objetivos específicos se encuentran: (1) analizar el papel de factores semántico-pragmáticos en la elección de uno por parte de los bogotanos; (2) observar si prima la interpretación referencial de uno asociada a un menor grado de impersonalidad o si se utiliza con matices distintos; (3) determinar si su empleo mantiene el mismo tipo de interpretación impersonal en relación con factores como la clase semántica del verbo, el discurso y la interacción de la entrevista; y (4) evidenciar su posible función como marca de las valoraciones del hablante, es decir, como mecanismo de subjetivización del discurso.

Al considerar la idea de que no todos los impersonales singulares poseen el mismo grado de versatilidad, especialmente cuando se intenta expresar subjetividad o apelar a un sentido de solidaridad en el marco de la interacción comunicativa, este estudio parte de la hipótesis de que en el habla de Bogotá se tiende a un empleo personal de uno: $\mathrm{Su}$ predominio puede apuntar más a una marca de subjetividad y menos a una estrategia de desfocalización, si la interpretación referencial de este impersonal se encuentra principalmente conectada con el yo-hablante. 


\section{Estado de la cuestión}

Aunque existen estudios de perspectiva histórica enfocados en el pronombre indefinido uno, Company y Pozas (2009: 1097) afirman que todavía faltan trabajos monográficos especializados: "la mayoría de las historias de la lengua y de gramáticas de referencia del español, sincrónicas y diacrónicas, sólo le dedica un brevísimo comentario de dos líneas [...] o nada". Esta escasez se extiende a los estudios de impersonalidad en el español colombiano. Los análisis empíricos enfocados en el funcionamiento de los impersonales singulares en corpus orales solo han empezado en la última década y han resaltado la influencia de los factores sociales mencionados en la introducción. Entre algunos trabajos, se cuenta con Montes Giraldo (1985), Arboleda (2001) y Dieck (en prensa). En las muestras recogidas entre 1958 y 1959 para el Atlas Lingüístico-Etnográfico de Colombia, Montes Giraldo (1985) registró la utilización impersonal de tú en oraciones como "El caimán como [...] y que tú no le hagas ruido, se deja rascar" (144) en el departamento de Bolívar, zona caribeña que adoptó el tuteo desde la época colonial por intensos contactos económicos y administrativos con España. En cuanto al uso de se impersonal, Arboleda (2001) se centra en las normas de concordancia (concordante y no-concordante) en una área dialectal andina de Colombia. Por último, Dieck (en prensa) analiza los porcentajes de impersonalización con se, uno y la segunda persona singular, e incluye además la tercera persona plural y la pasiva perifrástica. Demuestra que las formas más utilizadas en la ciudad de Medellín fueron uno (47.9\%), principalmente en función 
de sujeto lógico o agente, se (27.5\%) y la tercera persona plural (17.7\%). Registró muy pocos casos de empleo impersonal de la segunda persona singular (6.9\%: 22 de Ud., 4 de vos $y$ 0 de tú) y ninguno de la pasiva perifrástica (ser + participio).

\subsection{Acerca de la variabilidad referencial de uno}

Si bien las construcciones impersonales coinciden en un rango de usos, los estudios señalan que el empleo de una u otra forma refleja distintos grados de especificidad del agente. En el caso de uno, desde su aparición como impersonal y su avance en la segunda mitad del siglo XVI (Carrasco, 1988: 322), este se ha vinculado con la función de disminuir la responsabilidad del agente $y$, a la vez, ubicar la perspectiva del hablante en primer plano (Company y Pozas, 2009: 1196).

Por un lado, uno expresa una generalización conectada con la primera persona y la perspectiva del hablante (Haverkate, 1985: 19) y, por el otro, puede llegar a imprimir un valor de generalidad a un hecho u opinión particular (Company y Pozas, 2009: 1206). Esta característica de incluir al hablante y una situación más generalizadora, se observa en la experiencia relatada en el ejemplo (1), cuya lectura puede resultar o más definida al comunicar un evento personal, o más generalizadora al aludir la situación de todos aquellos que saben trabajar. Cuando la referencia puede extenderse a todos, parece más distanciada y objetiva (Kluge, 2010: 1118), una verdad incuestionable (Hollænder, 2002: 129):

(1) En cambio, yo trabajando, pues sé que ya después me puedo defender. Así me toque trabajar en cualquier oficio de empleada, 
pero uno sabiendo trabajar — que es lo más importante- en cualquier parte lo van a recibir a uno; así uno no sea un experto, pero uno sabiendo más o menos las cosas, lo reciben (Entrevista 9. Montes Giraldo et al., 1997: 202).

Como Company y Pozas (2009: 1206) indican, los casos de reducción completa de la referencia impersonal se reconocen cuando aparecen elementos textuales como el pronombre de primera persona o verbos en primera persona (me puedo defender, me toque trabajar). Esta reducción de la impersonalización, en la cual uno alude directamente a la situación del hablante, parece ser un fenómeno frecuente en Colombia, ya que en el análisis de Dieck (en prensa) del habla de Medellín, el 93 \% de los casos con uno ocurrió con referencia a la primera persona.

Un uso más impersonal se observa cuando el hablante asume (de manera empática) la perspectiva de cada integrante de un grupo determinado, cuando simula y se pone en el lugar del otro (Moltmann, 2006: 257-258). De igual manera, interpretar la referencia de uno con un grupo depende de la aparición de elementos textuales (Company y Pozas, 2009: 1206), como los que se observan en el ejemplo (2), es decir, su coaparición con verbos en primera persona plural:

(2) Pero tampoco tenemos las comodidades que tienen la gente, la mujer europea o la mujer norteamericana. Aquí tenemos... si no tenemos ayuda eh... de otras personas, es duro estar uno solo, y, además, pues ahora las mujeres somos un poco más conscientes sobre la educación de los hijos (Entrevista 13. Otálora y González, 1990: 178). 
En este fragmento, la entrevistada asume la perspectiva del grupo de mujeres colombianas, aunque no utiliza la forma femenina una. Es probable que la variación de género no sea frecuente en el habla colombiana, pues Dieck (en prensa) no registró casos en la muestra de Medellín.

\subsection{Aspectos coincidentes y divergentes en la interpretación referencial de uno, se y la segunda persona}

Según Carrasco (1988: 327), desde el siglo xvi se y uno ya comenzaron a tener funciones diferenciadas que en general se mantienen hasta hoy. El análisis de Ricós Vidal (2002: 946-956) sustenta que se (pasivo e impersonal) manifestaba objetividad y distanciamiento entre los interlocutores y el enunciado tanto en textos legales como expositivos. En cambio, uno se empleaba no solo para impersonalizar o desfocalizar el sujeto de verbos reflejos y pronominales, sino también para lograr un acercamiento a los interlocutores en los discursos argumentativos y doctrinales.

Para Vera Luján (1990: 93), precisamente esta conexión con el conocimiento y la experiencia del hablante distingue a uno de se. Se alcanza el máximo grado de distanciamiento (Briz, 2003) ya que expresa de manera mínima sus referentes (Siewierska, 2008b: 7). Su interpretación oscila entre lo genérico/cuasi-universal y lo corporativo, este último definido como un grupo socialmente determinado o un individuo representante del grupo (Casielles, 1996: 370). Es neutro con respecto a la orientación de la perspectiva (Haverkate, 1985: 19) e incluye "normas y reglas sociales, métodos establecidos, rutinas o, simplemente, una opinión generalmente 
aceptada" (Fernández, 2008: 224). Dicha objetividad se ilustra en el ejemplo (3), pues el hablante bogotano utiliza se para mostrar neutralidad o distancia de su opinión crítica acerca del grupo concreto de los gobernantes, con el fin de atenuar un evento negativo:

(3) Al gobierno obviamente le hizo falta bastante. Porque precisamente los padres de la patria, quienes legislan, pues tendrán que ver si se legisla para sí mismo o, por el contrario, se legisla para... para los desiguales. Y entonces cuando se legisla para los desiguales, se le aplica la ley a los más débiles (Entrevista 16, 2005).

No obstante, es posible designar con se la experiencia del hablante, con un menor grado de impersonalidad a la manera de uno, como ocurre en la siguiente narración del ejemplo (4):

(4) Bueno, yo por lo menos estoy haciendo segundo como le había dicho señorita; estoy haciendo segundo bachiller y... verdá que en ese colegio si se ven cosas raras, que muchacho loco, pero yo como voy a lo mío (Entrevista 22. Montes Giraldo et al., 1997: 483).

El empleo impersonal del pronombre de segunda persona singular ( $t u ́$ o $U d$.) constituye otra forma que en ciertos contextos puede alternar con uno. Para Fernández (2008: 223-224), tanto la segunda persona singular como uno expresan generalizaciones que parten de la experiencia del hablante y se proyectan hacia otras personas. Sin embargo, por las características de persona y número de la segunda 
persona, el hablante manifiesta un interés especial en el oyente y crea un ambiente de "participación empática" (Hollænder, 2002: 131), una relación intersubjetiva (Traugott, 2010). En el ejemplo (5), la entrevistada utiliza el tú impersonal al hablar de una situación que la afecta, tal vez con el ánimo de lograr la empatía de su interlocutor:

(5) Cuando un muchacho de estos te habla de lo que es su escuela... de por qué se la van a cerrar, de qué pasaría si le cerraran su escuelita, entonces tú empiezas a sentir algo que... que tú no vives, que no has visto, pero notas que otro lo vive, y empiezas como a sentirlo muy de cerca (Entrevista 15. Otálora y González, 1990: 209).

\subsection{Factores semántico-pragmáticos determinantes en el uso de los pronombres impersonales singulares}

Si bien la mayoría de los estudios sobre la impersonalización se ha concentrado en demostrar que cada forma indica un grado específico de impersonalidad, aquellos realizados mediante el análisis de corpus han destacado, además de la complejidad en el empleo de los impersonales singulares a nivel discursivo, la existencia de otros condicionamientos como la posición y actitud del hablante frente a la fuente de información y el tipo de información que transmite. Por ejemplo, Fernández (2008: 223-224), en su trabajo del habla culta de las ciudades de Madrid y Buenos Aires, propone la influencia de la característica de evidencialidad en su selección; es decir, el acceso al tipo de información disponible para el hablante: si la fuente de información es personal o si 
el acceso a la información es universal. Según este trabajo, el hablante generaliza con uno y tú a partir de su experiencia y con se mediante la inclusión de otras voces u opiniones generalmente aceptadas. Así, la elección de una forma u otra depende de la manera como el individuo decide presentar la información: más personal y privativa con uno y tú, más ajena y universal con se.

Algunos estudios empíricos, aunque enfocados en el análisis de la expresión del sujeto pronominal, han sugerido también la relevancia de la característica de impersonalidad en el empleo explícito de los pronombres tú, Ud. y uno (ej. Cameron, 1996: 89; Alfaraz, 2015:10). En las entrevistas orales realizadas con colombianos residentes en Miami, Hurtado (2005: 195), además de confirmar la influencia de esta característica en la expresión del pronombre de sujeto, muestra que uno es la forma utilizada de manera explícita con el peso probabilístico más alto (0.85). De acuerdo con sus resultados, dichos usos están determinados por la postura del hablante frente a lo enunciado, en cuanto a un contenido modal factual: los pesos probabilísticos con mayor efecto se encontraron en referencias a hechos ocurridos con anterioridad (0.63) que coincidían con la narración de eventos, en contraste con los pesos más bajos de las acciones que indicaban irrealidad o posibilidad.

Company y Pozas destacan que en tipos discursivos como la argumentación, la narración y el diálogo, el empleo de uno "[...] confiere a la generalización cierta subjetividad, derivada quizá por su identificación con el hablante [...]" (2009: 1207). El estudio de Gelabert-Desnoyer (2008: 421) ilustra esta función pragmática en el discurso parlamentario 
español, pues el pronombre uno se utiliza no solo para encubrir al hablante, sino también para aludir al interlocutor y a un grupo más general. En el caso del yo encubierto, el autor explica este empleo tanto por la necesidad de distanciarse como de evitar un uso redundante o "inelegante" de yo. En cuanto a la referencia intersubjetiva, sirve aun para atacar al interlocutor sin causar una reacción instantánea de su parte, conservando un comportamiento verbal educado.

La importancia de la narración de experiencias personales queda evidenciada en dos trabajos más. Flores-Ferrán (2009: 1821) observa la influencia del tipo de interacción y la polaridad del evento descrito en el análisis de uno y yo, en narraciones y entrevistas terapéuticas con 12 hispanos bilingües residentes de Nueva Jersey y Nueva York. Los individuos empleaban uno durante las entrevistas terapéuticas, cuando suministraban información neutral y sus enunciados estaban enmarcados por situaciones negativas. Bassa Vanrell (2013) advierte también la relación entre la referencia más incluyente del hablante y el uso predominante de uno en la narración de experiencias por parte de puertorriqueños y dominicanos, tanto en discursos orales como escritos (60\% Puerto Rico, 68\% República Dominicana).

Hasta aquí, los estudios previos muestran la complejidad y variabilidad en el empleo de uno, al apuntar a su versatilidad en la expresión de diferentes grados de interpretación impersonal y a su coincidencia con tú, $U d$. y se en algunos usos. Asimismo, los trabajos de corte semánticopragmático mencionan otros factores fundamentales en la elección de un impersonal, como la fuente de información, el tipo de discurso (narración de eventos personales, discur- 
so político) e interacción (entrevistas formales, entrevistas terapéuticas), y no solo el grado de generalización que se desee lograr.

Debido a que Fernández (2008) demuestra que el concepto de evidencialidad (la influencia del acceso a la fuente de información) es clave en la explicación de la impersonalidad, en el presente estudio se tendrá en cuenta este concepto, pero como un fenómeno deíctico que refiere al hablante (Haßler, 2010: 243) y que ocurre en contextos altamente subjetivos como con verbos de contenido emotivo y volitivo, narraciones de experiencias y situaciones comunicativas de cercanía: se considerará como una elección consciente del hablante para dirigir la atención hacia sí mismo, sobre todo cuando menciona eventos experimentados de primera mano (Aikhenvald, 2004: 237). De igual manera, se intentará vincular los términos de subjetividad e intersubjetividad con base en Traugott (2010: 30-33), con el fin de analizar el pronombre uno como una posible marca lingüística de la posición y perspectiva del hablante frente a su propia experiencia e, incluso, de la expresión de su conciencia o conocimiento de las actitudes y creencias del oyente y de los otros. Así, se examinará la subjetividad como la conexión con el hablante, sus creencias y actitudes, y la intersubjetividad como la relación con el oyente, para analizar la probabilidad de que en el contexto bogotano esté ocurriendo un tipo de reanálisis semántico de la característica de impersonalidad de uno en favor de la interpretación más ligada a yo: "la reinterpretación de las relaciones [...] semánticas que una forma o construcción contrae y supone un cambio en su estatus categorial” (Company, 2010: 37). 


\section{Metodología}

\subsection{Población y muestra}

La muestra oral comprende tres grupos de entrevistas que concuerdan con tres periodos. Si bien el número de entrevistas y sus modalidades de interacción no es exactamente la misma en cada periodo, las incluimos en este trabajo en un intento de observar si a través de los años se detectaba este predominio de uno o si, por el contrario, se trata de un fenómeno reciente. La muestra 1 constituye un punto de referencia sobre la posible norma o las formas de prestigio, al incluir los usos impersonales de la clase educada. Las muestras 2 y 3 aportan valiosa información sociolingüística por contener todas las variedades diastráticas y tratarse de interacciones más espontáneas:

1. Sesenta diálogos recogidos de 1972 a 1984 y publicados por el Instituto Caro y Cuervo (Otálora y González, 1990), parte del proyecto de Estudio de la norma lingüística culta de las principales ciudades de Iberoamérica y de la Península Ibérica. De esta muestra incluimos tres modalidades: diálogos entre informante y encuestador, diálogos entre dos informantes y conversaciones espontáneas, es decir, el 90\% de la muestra. No incluimos las elocuciones formales como clases, conferencias y discursos. Cada entrevista tuvo una duración de 30 minutos. Los entrevistados eran en su mayoría bachilleres y profesionales pertenecientes a las clases media y alta, cuyas edades oscilaban entre 25 y 84 años. 
2. Treinta relatos semilibres recolectados entre 1990 y 1992 por los investigadores del Instituto Caro y Cuervo para el proyecto de El español hablado en Bogotá (Montes Giraldo et al., 1997). Los relatos y conversaciones de diferente grado de formalidad fueron recogidos a través de métodos sociolingüísticos, con ayuda de un cuestionario y en las casas de los participantes. Cada entrevista duró aproximadamente una hora.

3. Treinta conversaciones recopiladas en dos temporadas en Bogotá, una en 2005 y la otra en 2008. Los participantes fueron contactados partiendo de las redes de interacción social de la autora (originaria de Bogotá). La interacciones se realizaron en las casas de los entrevistados con un tiempo promedio de 45 min. Se trataba de conversaciones con diversos grados de interacción y familiaridad entre el investigador y dos entrevistados, las cuales incluían relatos de experiencias personales, temas de opinión y situaciones hipotéticas.

Las muestras 2 y 3 cuentan con participantes de estratos bajo, medio y alto, con diversos niveles de ocupación y grados de escolaridad. Las edades de los participantes oscilaban entre los 18 y los 80 años en el momento de la entrevista. La mayoría de ellos había nacido en Bogotá. Aquellos que llegaron a la ciudad durante su infancia, procedían principalmente de zonas rurales próximas, en especial de los departamentos de Cundinamarca y Boyacá. 


\subsection{Delimitación del contexto variable}

Desde una perspectiva semántica y de acuerdo con el contexto textual y discursivo, se eligieron oraciones cuyo sujeto podía interpretarse con diversos grados de generalización (yo oculto, nosotros, referencia general). Se codificaron 4,792 ejemplos con el indefinido uno, la construcción con se y los usos impersonales de tú y usted, los cuales constituyen la variable dependiente. Se parte de estas construcciones impersonales ya que, como se mencionó en la introducción, este estudio intenta analizar esa característica de versatilidad que permite referir/ocultar al hablante, integrar al oyente y a una tercera persona, y denotar a la humanidad entera. Para distinguir los casos dudosos de impersonalización con tú y $U d$., se consideraron inductores de generalidad como la imperfectividad de la acción, la clase semántica del verbo y activadores del carácter genérico como siempre y en situaciones de ese tipo (Hernanz, 1990: 157; Muñiz Cachón, 1998: 76).

En las muestras primó la utilización de uno con función de sujeto (81.6\%), tanto de manera expresa como tácita (ej. 6a), y como sujeto de infinitivo (ej. 6b) o de gerundio (ej. 6c). Además, se codificaron los casos de este pronombre en función de objeto directo (ej. 6d, 0.4\%) o indirecto (ej. 6e, $3 \%)$. Si bien con ciertos verbos pronominales y construcciones reflexivas el uso de uno y la segunda persona resulta obligatorio (Fernández, 2008: 230) y el empleo de se, agramatical, se incluyeron dichos verbos porque uno podía alternar con $U d$. (ej. 6 f). De esta manera, se logró considerar que esta limitación estructural no se relacionaba con el alto porcentaje de uno por parte de los bogotanos, ya que estos 
casos solo constituyeron un 15\% del total de la muestra. Es necesario también resaltar que solo ocurrieron 6 casos del uso de una, es decir, con variación de género, y que como en el ejemplo (ej. $6 \mathrm{~g}$ ) coocurrieron con uno.

Ejemplos extraídos de la muestra de Montes Giraldo et al., (1997):

(6) a. Bueno, a veces uno ni se entera de las cosas, porque uno está limitado a la casa y los hijos y... de ahí no se entera de nada más (Entrevista 22: 484).

b. Pero tampoco uno dejarse encaprichar (Entrevista 7: 150).

c. Pero uno sabiendo trabajar, que es lo más importante [...] (Entrevista 9: 202).

d. Cuando lo invitan a uno a un matrimonio (Entrevista 9: 193).

e. Digamos, eso a uno le duele mucho (Entrevista 9: 209).

f. [...] porque desafortunadamente va pasando el tiempo y... pues, le dedica... $\underline{\text { se }}$ lo dedica uno a otras cosas (Entrevista 4 . Otálora y González, 1990: 58).

g. Uno ve tantos matrimonios, es que el daño está por encima. Una ve tantos matrimonios que se han separado y se casan dizque por lo civil (Entrevista 24: 533).

Se analizaron cinco variables independientes que proporcionan información sobre el uso subjetivo de uno. Las variables lingüísticas comprenden aquellas que marcan la perspectiva del hablante como la interpretación referencial, la clase semántica del verbo y el tipo de discurso. La variable pragmática de clase de entrevista contiene factores contextuales decisivos en la variación de los impersonales singulares, como la información sobre la relación entre los individuos interactuantes; el pronombre de tratamiento usado en 
la entrevista es un posible índice del nivel de participación e implicación del hablante.

3.2.1. Interpretación referencial. Esta variable está constituida por los diferentes grados de impersonalidad mencionados en la introducción, de la siguiente manera:

1. El referente se identifica con el hablante (yo). En el ejemplo (7), el pronombre de primera persona y el verbo ser en primera persona coaparecen con uno, lo cual habilita a clasificarlo como un caso de referencia a la experiencia del yo-hablante:

(7) Yo mostraría las dos realidades. Soy una persona partidaria de mostrar lo que uno es (Entrevista 22, 2008).

2. El hablante alude a su experiencia y la de otros, entre quienes podía incluir implícitamente al interlocutor (nosotros), como en el ejemplo (8):

(8) [...] porque cuando nos vinimos para acá, era la primera vez que vivíamos en el sur, para uno era tremendo (Entrevista 11, Montes Giraldo et al., 1997: 237).

3. El hablante menciona situaciones generales y hasta referencias aplicables a cualquiera o a un grupo de individuos que excluye al hablante y al oyente (general). En el ejemplo (9), dicha exclusión se introduce con se impersonal y la tercera persona plural todos:

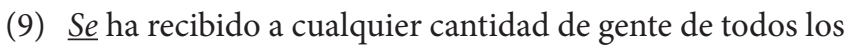

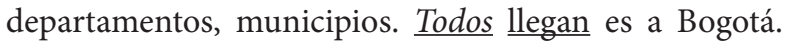
Sea lo que sea Bogotá, uno no se muere de hambre, el que tiene es porque quiere (Entrevista 11, 2005). 
Además del análisis del sujeto expresado en las oraciones previas, se tuvo en cuenta que los casos de primer y segundo grado suceden en un tiempo concreto, y los del tercer grado, sin referencia temporal específica (Siewierska, 2008b: 9).

3.2.2. Clase semántica del verbo. La clasificación consta de verbos cuyo contenido léxico puede reflejar la experiencia directa del hablante en términos de evidencia probabilidad, evaluación e inseguridad, y puede promover una interpretación referencial más o menos impersonal. Comprende 7 categorías basadas en las siguientes distinciones semánticas:

1. Comunicación. Principalmente se trataba de los verbos decir y hablar:

(10) Pero cuando uno tiene un trabajo malo y de pronto ve uno la oportunidad y dice, uy, aquí me cuadro (Entrevista 23,2008 ).

2. Percepción física. Se incluyen los verbos de percepción visual y auditiva como ver, escuchar, oír y mirar. En el ejemplo (11), el hablante utiliza ver para mencionar su experiencia directa del evento:

(11) Aquí en el barrio pues uno ve las cosas y... colabora uno con todos los del barrio, con las alarmas (Entrevista 2, 2005).

3. Percepción mental. Se clasificaron los verbos de conocimiento, opinión o pensamiento como saber, aprender, darse cuenta y creer: 
(12) Y uno aprende en una palabra, por ejemplo, que si los muchachos lo critican a uno, entonces uno aprende a corregir eso y ya sabe (Entrevista 21. Montes Giraldo et al., 1997: 470).

4. Volitivos. Se consideraron aquí los verbos que indican influencia y deseo, y modales de obligación y permiso como deber y poder, los cuales favorecen la lectura genérica (Company y Pozas, 2009: 1202) como en el ejemplo (13):

(13) Yo creo que todos viven como yo, en conflicto. Es un conflicto permanente porque no está uno trabajando en lo que uno quiere [...] Eh... está uno todo el día haciendo una cosa y pensando que debería uno estar practicando (Entrevista 6. Otálora y González, 1990: 93).

5. Emoción. Comprende los verbos de actitud y sentimiento como sentir y preocuparse:

(14) Cuando uno tiene una obra en dedos, la sabe, y le ha llegado al fondo, la entiende, la comprende y la siente, yo creo que siente uno una alegría... (Entrevista 6. Otálora y González, 1990: 76).

6. Estado. Se refiere principalmente a ser, estar y tener:

(15) Pero era que el empleado público era mucho más sacrificado que hoy, y uno también era más sacrificado [...] (Entrevista 18. Otálora y González, 1990: 241).

7. Dinámicos. Además de verbos como dar y hacer (ejemplo 16), vinculados con un evento llevado a cabo por el hablante, incluye los verbos ir y venir, salir y llegar, llevar y traer (ejem- 
plo 17) que proporcionan información acerca de la ubicación del hablante:

(16) A veces uno... pues no hace la lista de las cosas que tiene que hacer y no le pone orden... (Entrevista19. Montes Giraldo et al., 1997: 418).

(17) En Girardot, pues ya llegaba uno y se bajaba uno a buscar hospedaje, ¿no? y $\underline{\text { irse }}$ uno a conocer el río Magdalena [...] (Entrevista 18. Montes Giraldo et al., 1997: 410).

3.2.3. Tipo de discurso. Puesto que Company y Pozas (2009: 1203-1207) observan que uno confiere cierta subjetividadidentificación con el hablante en tipos discursivos como la argumentación y la narración y, además, los contextos condicionales son propicios para la utilización de uno, este grupo incluye:

1. Narración (experiencias personales)

2. Exposición y argumentación (hechos generales)

3. Conjeturas e hipótesis (situaciones hipotéticas, proyección de acciones).

3.2.4. Clase de entrevista. Se considera el grado de interacción, el nivel de formalidad de la entrevista y la relación de los interactuantes de la siguiente manera:

1. Poca interacción: Ante la pregunta del entrevistador, el entrevistado desarrolla respuestas largas, casi una 
exposición. Hay poca interacción y seguimiento de las ideas por parte del entrevistador. El nivel de formalidad es muy alto y la relación entre los participantes, asimétrica.

2. Individual formal: Ocurre más interacción de preguntas y respuestas entre el entrevistador y el entrevistado. No obstante, la utilización formal de $U d$. y títulos (ej. doctor) como formas de tratamiento, plantea distancia entre los individuos.

3. Individual espontánea: La interacción entrevistadorentrevistado es informal. Se percibe un trato de confianza entre ellos.

4. Diálogos entre conocidos: Interacción informal entre conocidos sin intervención del entrevistador. Máximo trato de confianza e identificación del uno con el otro.

3.2.5. Pronombre de tratamiento. En cuanto a la conexión entre las formas de tratamiento típicas de una región y la preferencia de un pronombre impersonal, DeMello (2000) sugiere que el mayor porcentaje de generalización con uno y se ocurre cuando los interactuantes se tratan de usted. Sin embargo, Hurtado (2012) comprobó un predominio de uno tanto en variedades tuteadoras como ustedeadoras de Colombia, y Guirado (2011) en la variedad tuteadora de Caracas. Por eso, y debido a que en Bogotá se utiliza tanto $U d$. como tú como formas de tratamiento (Montes Giraldo, 1982), se clasificó el pronombre utilizado por el entrevistado durante la entrevista con el fin de explorar la posibilidad de que uno ocurra con mayor frecuencia cuando los participantes se tratan de usted por un aparente grado de formalidad (ejem- 
plo 18), o tal vez cuando se tratan de tú, por ser una situación de confianza y en la cual se utiliza uno como una forma más coloquial (ejemplo 19):

(18) [...] pongamos, que les va a creer uno a los guerrilleros, que les va a creer uno. Fíjese que ellos están firmando la paz y por detrás están otra vez haciéndose de las suyas (Entrevista 24. Montes Giraldo et al., 1997: 543).

(19) Bueno, me has dado una oportunidad que muy raras veces la tiene uno, de recordar esas épocas tan agradables de la ciudad de Bogotá (Entrevista 29. Otálora y González, 1990: 385).

\section{Resultados y análisis}

\subsection{Distribución de las construcciones impersonales en la muestra}

Para dilucidar qué impersonales predominaban de acuerdo con el criterio de la interpretación referencial, se examinó el contexto lingüístico donde aparecían y se consideraron los diferentes grados de impersonalización mencionados en el apartado anterior. Conforme con los porcentajes totales del cuadro 1, en Bogotá predominan las formas uno y se $(51.1 \%$ y $43.5 \%)$ frente a los usos de tú (2.4\%) y Ud. (3\%). Fundamentalmente, los participantes utilizan se a medida que el grado de generalidad aumenta $(76.6 \%$ para mencionar ideas aplicables a cualquiera, un sentido genérico) y uno en 
cuanto el referente es el hablante (83.3\%). Uno y se presentan casi los mismos porcentajes al remitir a nosotros $(49.1 \%$ y $47.1 \%$ ), aunque se advierte que uno no solo expresa un sentido cercano a yo, sino también una pluralidad implícita cuando el hablante asume la postura de un grupo específico (nosotros).

Cuadro 1. Distribución de los singulares impersonales según su interpretación referencial

\begin{tabular}{|c|c|c|c|c|c|c|c|c|}
\hline \multirow[b]{2}{*}{ Impersonal } & \multicolumn{2}{|c|}{ Yo } & \multicolumn{2}{|c|}{ Nosotros } & \multicolumn{2}{|c|}{ General } & \multicolumn{2}{|c|}{ Total } \\
\hline & $\mathbf{N}$ & $\%$ & $\mathbf{N}$ & $\%$ & $\mathbf{N}$ & $\%$ & $\mathbf{N}$ & $\%$ \\
\hline Uno & 1546 & 83.3 & 659 & 49.1 & 245 & 15.4 & 2450 & 51.1 \\
\hline $\mathrm{Se}$ & 230 & 12.4 & 631 & 47.1 & 1223 & 76.6 & 2084 & 43.5 \\
\hline Tú & 49 & 2.6 & 24 & 1.8 & 43 & 2.7 & 116 & 2.4 \\
\hline Usted & 30 & 1.6 & 27 & 2.0 & 85 & 5.3 & 142 & 3.0 \\
\hline Total muestra & 1855 & 38.7 & 1341 & 28.0 & 1596 & 33.3 & 4792 & \\
\hline
\end{tabular}

Estos resultados pueden constituir un primer indicio del uso de uno para lograr una subjetivización del discurso al ubicar al hablante e involucrar al oyente y a otros en un contexto determinado, función analizada por Kluge (2010: 1118-1125) como característica de la impersonalización con tú y Ud. (corpus de 2005 del habla madrileña), sobre todo en contextos donde existe una relación cercana entre los interactuantes. En cuanto a la escasez de los impersonales de segunda persona en Bogotá, probablemente estos pronombres no indican claramente grados de respeto o distancia social (Head, 1978: 167) como sí lo puede reflejar el empleo de uno y se en las interacciones en Bogotá.

El vínculo entre los usos impersonales con la distancia o cercanía que expresan los pronombres de tratamiento 
queda refutado en los resultados del cuadro 2. Los porcentajes del pronombre utilizado por el informante para dirigirse a su interlocutor en el momento de la entrevista revelan que quienes ustedearon y tutearon a su interlocutor utilizaron tanto uno (54.1\% y $44.8 \%$ ) como se (42.1 y $46.4 \%$ ) casi en la misma dirección, pero los tuteadores emplearon casi todos los casos del impersonal tú (7.1\% de 7.3\%). Mas bien, sugieren que aun cuando normalmente las formas de tratamiento $U d$. y tú varían según la función socio-pragmática de los interactuantes, este aspecto parece no determinar la preferencia por cierto impersonal.

Cuadro 2. Porcentaje de impersonales singulares en relación con el pronombre de tratamiento utilizado por el entrevistado para dirigirse a su interlocutor

\begin{tabular}{|c|c|c|c|c|c|c|c|c|c|c|}
\hline \multirow[t]{2}{*}{ Tratamiento } & \multicolumn{2}{|c|}{ Uno } & \multicolumn{2}{|c|}{ Se } & \multicolumn{2}{|c|}{ Tú } & \multicolumn{2}{|c|}{ Usted } & \multicolumn{2}{|c|}{ Total } \\
\hline & $\mathbf{N}$ & $\%$ & $\mathbf{N}$ & $\%$ & $\mathbf{N}$ & $\%$ & $\mathbf{N}$ & $\%$ & $\mathbf{N}$ & $\%$ \\
\hline Usted & 1770 & 54.1 & 1379 & 42.1 & 8 & 0.2 & 117 & 3.6 & 3274 & 68.3 \\
\hline Tú & 680 & 44.8 & 705 & 46.4 & 108 & 7.1 & 25 & 1.6 & 1518 & 31.7 \\
\hline
\end{tabular}

Por último, el cuadro 3 proporciona información sobre la posible norma de la clase educada entrevistada de 19721984, frente a los usos de los dos periodos siguientes, los cuales incluyen individuos de todos los estratos sociales. En términos generales, a pesar de que los porcentajes de uno y se son los más altos en cada periodo, se detecta el aumento de uno en los dos últimos (de 42.7 a 56.4 y 56.2\%), una expansión de los dominios de uno a situaciones referenciales que en el primer periodo eran propias de se. Como las entrevistas del primer periodo provenían del proyecto sobre el 
habla culta de Bogotá, es viable la conexión del empleo de se con un mayor grado de educación y con las experiencias intelectuales de la gente de un estrato más alto. Cuervo (1914: 224) ya señalaba la utilización innecesaria de $s e$ en vez de uno, hombre o nosotros, como resultado de la influencia de la lectura de libros franceses.

Cuadro 3. Porcentajes de uso de los impersonales singulares según la fecha de la entrevista:

Incremento de la frecuencia de uno

\begin{tabular}{|l|r|c|c|c|c|c|c|c|}
\hline \multirow{2}{*}{ Fecha entrevista } & \multicolumn{2}{|c|}{ Uno } & \multicolumn{2}{c|}{ Se } & \multicolumn{2}{c|}{ Ud. } & \multicolumn{2}{c|}{ Tú } \\
\cline { 2 - 9 } & $\mathbf{N}$ & $\mathbf{\%}$ & $\mathbf{N}$ & $\mathbf{\%}$ & $\mathbf{N}$ & $\mathbf{\%}$ & $\mathbf{N}$ & $\%$ \\
\hline $1972-1984$ & 779 & 42.7 & 950 & $\mathbf{5 2 . 1}$ & 35 & 1.9 & 61 & 3.3 \\
\hline $1990-1992$ & 1384 & $\mathbf{5 6 . 4}$ & 991 & 40.4 & 53 & 2.2 & 28 & 1.1 \\
\hline $2005-2009$ & 287 & $\mathbf{5 6 . 2}$ & 143 & 28.0 & 54 & $\mathbf{1 0 . 6}$ & 27 & $\mathbf{5 . 3}$ \\
\hline Total muestra & $\mathbf{2 4 5 0}$ & $\mathbf{5 1 . 1}$ & $\mathbf{2 0 8 4}$ & $\mathbf{4 3 . 5}$ & $\mathbf{1 4 2}$ & $\mathbf{3 . 0}$ & $\mathbf{1 1 6}$ & $\mathbf{2 . 4}$ \\
\hline
\end{tabular}

No obstante, una tabulación cruzada con la variable de interpretación referencial mostró que tanto se como uno fueron utilizados con referencias diferenciadas en este primer periodo: generales-se (94\%), nosotros-se (54\%) y youno (83\%). Puesto que $65 \%$ de los 779 casos de uno se trataba de narración de experiencias personales, se infiere que la interpretación más común de uno en los años setenta ya tendía hacia un menor grado de impersonalidad, como ilustra el ejemplo (20):

(20) Ahora cuando fui a Ibagué estuve muy contenta, la vida mía fue muy sabrosa, sino que el cambio a veces le da 
como ... a uno neura. Pensar uno que no es lo mismo, ¿no? (Entrevista 38. Otálora y González, 1990: 514).

De igual manera, es interesante que en los años dos mil, el porcentaje de se disminuye a $28 \%$ : de la neutralidad expresada con la construcción se, la que comúnmente reduce el protagonismo del hablante, del oyente $\mathrm{u}$ otros agentes (Martínez-Linares, 2009: 232), se pasa a la inclusión del agente, mediante uno, y posiblemente el aumento del interés hacia el receptor, con el incremento de los impersonales $U d$. y tú (10.6\% y $5.3 \%$, respectivamente). A pesar de que se vislumbran diferencias en el uso impersonal a través de los años, la falta de una muestra más equitativa para cada periodo no permite analizar más en detalle esta variable temporal en este estudio.

\subsection{Resultados del análisis de regresión múltiple, Goldvarb}

Puesto que de los 4,792 ejemplos solo 258 eran de casos de $t u ́$ y $U d$. generalizadores, para realizar los cómputos binomiales con el programa estadístico de regresión múltiple Goldvarb, se consideraron únicamente los casos de uno y se como variables dependientes. Este programa suministra evidencia en tres niveles: la significatividad estadística de las variables al nivel 0.05; la magnitud del efecto o rango, qué variable es más o menos determinante; la jerarquía de la influencia, precisada por el orden de los pesos probabilísticos Goldvarb dentro de cada grupo de variables (Tagliamonte, 2006: 235). Asimismo, suministra los pesos de relevancia probabilística para cada factor comprendido 
en las variables independientes: el número más cercano a 0.99 indica una influencia fuerte, a 0.00, falta de efecto, y a 0.50, neutralidad.

Tras el análisis binomial (Step-up/step-down), el programa estadístico escogió como significativas (al nivel 0.05) las variables de interpretación referencial, tipo de discurso, clase semántica del verbo y clase de entrevista. Eliminó la variable de pronombre de tratamiento utilizado por el entrevistado para dirigirse a su interlocutor por no mostrar una influencia significativa en los datos. Así, se confirma la falta de efecto sugerida en los porcentajes generales del cuadro 2.

\subsection{Influencia de los factores semántico-pragmáticos}

En cuanto al orden de los grupos de variables según la magnitud de su efecto (rango), el análisis de regresión múltiple reveló que los criterios de interpretación referencial y clase semántica del verbo ejercieron el efecto más significativo (ver en el cuadro 4: rango 75 y 55). El rango se calcula con base en la diferencia numérica entre el peso probabilístico más bajo y el más alto de cada grupo (cuadro 4).

Conforme con los pesos probabilísticos de la variable de interpretación referencial, se favorece uno cuando la referencia está vinculada con la experiencia del hablante (peso probabilístico 0.85 ), lo cual podría explicarse como una estrategia para evitar el abuso de yo, o como un mecanismo para establecer cierta distancia en lo relativo a su propia experiencia. Sin embargo, el hecho de que el pronombre uno haya sido utilizado de manera explícita en 1,297 de los 1,776 
Cuadro 4. Influencia de los factores semánticopragmáticos:

Resultados del análisis de regresión múltiple, Goldvarb

\begin{tabular}{|l|r|r|r|r|r|r|r|}
\hline \multirow{2}{*}{\multicolumn{1}{|c|}{ Variables }} & \multicolumn{3}{|c|}{ Uno } & \multicolumn{2}{c|}{ Se } & \multicolumn{2}{c|}{ Total } \\
\cline { 2 - 7 } & $\mathbf{N}$ & \% & Peso & N & $\%$ & N & $\%$ \\
\hline Inter. referencial & & & & & & & \\
Yo & 1546 & 87.0 & $\mathbf{0 . 8 5}$ & 230 & 13.0 & 1776 & 39.2 \\
Nosotros & 659 & 51.1 & 0.50 & 631 & 48.9 & 1290 & 28.5 \\
General & 245 & 16.7 & 0.10 & 1223 & 83.3 & 1468 & 32.4 \\
Rango: 75 & & & & & & & \\
Clase semántica & & & & & & & \\
del verbo & & & & & & & \\
Percepción física & 159 & 47.9 & 0.28 & 173 & 52.1 & 332 & 7.3 \\
Comunicación & 124 & 46.1 & 0.32 & 145 & 53.9 & 269 & 5.9 \\
Dinámicos & 1036 & 47.0 & 0.46 & 1169 & 53.0 & 2205 & 48.6 \\
Volitivos & 327 & 48.7 & 0.42 & 344 & 51.3 & 671 & 14.8 \\
Mentales & 309 & 65.2 & $\mathbf{0 . 5 7}$ & 165 & 34.8 & 474 & 10.5 \\
Emotivos & 83 & 87.4 & $\mathbf{0 . 8 2}$ & 12 & 12.6 & 95 & 2.1 \\
Estado & 412 & 84.4 & $\mathbf{0 . 8 3}$ & 76 & 15.6 & 488 & 10.8 \\
Rango: 55 & & & & & & & \\
Tipo de discurso & & & & & & & \\
Narración & 1346 & 62.2 & $\mathbf{0 . 5 2}$ & 818 & 37.8 & 2164 & 47.7 \\
Hechos generales & 852 & 42.6 & 0.43 & 1150 & 57.4 & 2002 & 44.2 \\
Hipótesis y conj. & 252 & 68.5 & $\mathbf{0 . 7 5}$ & 116 & 31.5 & 368 & 8.1 \\
Rango: 32 & & & & & & & \\
Clase entrevista & & & & & & & \\
Individual formal & 885 & 54.5 & 0.40 & 738 & 45.5 & 1623 & 35.8 \\
Indiv. espontánea & 753 & 60.6 & $\mathbf{0 . 6 2}$ & 489 & 39.4 & 1242 & 27.4 \\
Diálog. conocidos & 665 & 54.1 & $\mathbf{0 . 5 5}$ & 555 & 45.9 & 1210 & 26.7 \\
Poca interacción & 157 & 34.2 & 0.40 & 302 & 65.8 & 459 & 10.1 \\
Rango: 21 & & & & & & & \\
\hline
\end{tabular}

Nota: Significatividad $=0.000$. Chi-square total $=2214.5778$. Chi-square/cell $=1.8594$ Input: 0.58 Logaritmo de verosimilitud: -1883.296 
casos, puede promover en el oyente una lectura más directamente asociada con el hablante. Así, la expresión explícita de uno funcionaría como en el caso de la primera persona singular: para incrementar la atención en el referente del sujeto (Posio, 2011). Esta tendencia se detectó en los tres tipos de interpretación referencial: de los 2,450 casos de uno, 2,033 ocurrieron de manera expresa y 417, tácita. La mención explícita de este pronombre indefinido podría estar induciendo a una interpretación más vinculada con el yo, principalmente en función de sujeto, como en el ejemplo (21):

(21) - ¿A usted no le ha favorecido cuando va así vestido, como que se siente más protegido, que no le va a pasar nada?

-Pues, a veces uno piensa que de pronto uno se siente más protegido, pero no creo porque uno es un blanco. Mejor dicho, si, pues, uno dice, o sea, lo ven uniformado a $\underline{u n o}$ y de una vez lo señalan a $\underline{u n o}$. Y como está la situación hoy en día, ¿sí? Entonces mejor uno andar como, como de civil ¿sí? Porque uno así, uniformado, es un blanco (Entrevista 15, 2005).

Cuando uno aparece en combinación con adjetivos posesivos promueve aún más esas situaciones en las cuales el hablante añade énfasis (Orozco, 2012: 220). De acuerdo con Company y Pozas (2009: 1204), se trata de una relación de correferencia propia del impersonal uno, con la cual mantiene persistencia referencial. En el ejemplo (22), además de que se observa un continuo empleo de uno, se combina con el posesivo su: 
(22) Lo que pasa es que uno como que trata de andar con cuatro ojos, y pues de pronto si irse en compañía de alguna persona, pues uno se acompaña, igual a la avenida y eso. Y no andar de noche en la calle, creo yo que eso es lo mejor...para uno cuidar su integridad (Entrevista 10, 2005).

En cuanto a se, los entrevistados no lo emplean para explicar aspectos de su comportamiento ni de los otros, probablemente porque, como Casielles (1996: 373) ya había sugerido, esta construcción muestra una restricción que implica la irrelevancia del agente. Los resultados de Fernández (2008: 226) van también en esta dirección, pues de 277 ejemplos de construcción con se solo 6 se combinaron con la primera persona.

La segunda variable con el efecto más importante es la clase semántica del verbo (rango 55). Según los pesos probabilísticos, los verbos de estado, de emoción y los mentales promueven uno $(0.83,0.82$ y 0.57$)$, verbos que reflejan la subjetividad del hablante. Como se observa en el ejemplo (23), su contenido léxico indica estados, sentimientos y opiniones vinculadas con la expresión de yo:

(23) No, y además, y aquí en la universidad, yo nunca sentía que esto era como normal. Que decía, bueno, uno sí siente en la gente, la aprensión de la gente. Entonces, eso trata como de afectarte a ti, el ambiente (Entrevista 27, 2008).

Mediante la utilización de uno con los verbos de percepción mental como saber, conocer, entender y darse cuenta 
(ejemplo 24), el individuo manifiesta su grado de conocimiento de los eventos. De igual manera, evalúa y manifiesta probabilidad con verbos de opinión como creer y pensar.

(24) Entonces como uno no sabe lo que es el hambre, no puede entender que alguien pueda matar por hambre, ¿cierto? (Entrevista 15. Otálora y González, 1990: 203).

Es posible confirmar esta idea en el cuadro 5, donde se cruza la variable de clase semántica del verbo con la de interpretación referencial, pues el predominio de uno ocurre principalmente con los verbos estativos, mentales y emotivos, los cuales se asocian con un estado sicológico (ejemplo 23) o un proceso cognitivo del yo-hablante. Precisamente, verbos como estar, ser, entender y creer han mostrado un empleo de alta frecuencia con pronombres de primera persona singular (Posio, 2011: 784). No obstante, según el cuadro cruzado 5, es más relevante la variable de interpretación referencial que la clase semántica del verbo, pues predomina la interpretación de uno-yo en todos los tipos de verbos. Mientras que el individuo elige uno para hablar de sus propias experiencias, creencias, emociones y actitudes, se mueve entre un contenido objetivo y subjetivo observable en el significado léxico de los verbos. Cuando la referencia se vincula a nosotros, se distingue el mismo empleo de uno y verbos de estado, emotivos y mentales ( $83 \%, 81 \%$ y $60 \%$ ); con alusiones más generales, solo con los emotivos y de estado (64\% y $50 \%)$. 
Cuadro 5. El empleo de uno según la clase semántica del verbo y su interpretación referencial (cuadro cruzado)

\begin{tabular}{|l|c|c|c|}
\hline Clase semántica del verbo & Yo & Nosotros & General \\
\hline Percepción física & $\mathbf{7 4}$ & 35 & 9 \\
\hline Comunicación & $\mathbf{7 8}$ & 45 & 8 \\
\hline Dinámicos & $\mathbf{9 1}$ & 48 & 12 \\
\hline Volitivos & $\mathbf{7 7}$ & 44 & 21 \\
\hline Mentales & $\mathbf{8 8}$ & $\mathbf{6 0}$ & 21 \\
\hline Emotivos & $\mathbf{9 5}$ & $\mathbf{8 1}$ & $\mathbf{6 4}$ \\
\hline Estado & $\mathbf{9 7}$ & $\mathbf{8 3}$ & $\mathbf{5 0}$ \\
\hline
\end{tabular}

Con respecto a los verbos de percepción física y comunicación, cuando el hablante menciona las experiencias que percibe de manera directa, favorece el empleo de uno (74\%), como en el ejemplo (25):

(25) Yo he estado en Cali dos oportunidades y uno ve que hay mucha plata. Se mueve mucho dinero, y las mujeres allá se visten super bien. Y uno ve cualquier sardinita de cualquier estrato que más o menos uno se dé cuenta, y la gente anda bien vestida (Entrevista 18, 2005).

Sin embargo, los porcentajes más bajos de uno ocurren en cuanto se trata de referencias a un nosotros (35\% y 45\%) o a referencias más generalizadoras ( $9 \%$ y $8 \%$ ). Al parecer, uno no rinde en la expresión de la intersubjetividad y objetividad que se desea con este tipo de verbos. Estos resultados concuerdan con los de Fernández (2008: 228), pues en su estudio se ocurrió principalmente con verbos asociados a un carácter más objetivo tales como ver y oír, y cuya fuente de conocimiento no era privativa sino universal. 
La tercera variable más importante es el tipo de discurso (rango 32). Los pesos probabilísticos indican (cuadro 4) que el hablante opta por uno (0.75) cuando formula hipótesis, ejerce un efecto neutro al narrar (0.52), y lo desfavorece en la mención de hechos generales (0.43). La selección de uno se encuentra condicionada por los tipos discursivos más irreales porque tal vez el hablante asume la perspectiva de cada persona en un contexto determinado: cuando simula ser el otro en situaciones hipotéticas, basado en su conocimiento de los eventos y no en su experiencia directa. Se ilustra este uso en el ejemplo (26), ya que el entrevistado habla del desempleo con un razonamiento sobre la situación general padecida en Colombia y que además posiblemente afecta al hablante y al oyente:

(26) Ya es como cuestión de actitud. Si uno se echa la pena, pues ahí sí no hay nadie que lo saque. Pero no, en Colombia sí hay oportunidades lo que pasa es que uno no las sabe, o no las ve o no las sabe aprovechar (Entrevista 6, 2005).

No obstante, el cruce con la variable de interpretación referencial (cuadro 6) permite observar que el vínculo unoyo $(87 \%, 85 \%, 90 \%)$ continúa siendo más determinante que el tipo de discurso. Cuando se alude a nosotros, el porcentaje de uno incrementa al exponer y argumentar (59\%) y al lanzar conjeturas e hipótesis (65\%), lo cual puede apuntar a la extensión de la funcionalidad de uno:

(27) Los que creemos en Dios, ante Dios no es matrimonio si uno no forma un hogar, tiene que ser ante Dios y ante 
la sociedad (Entrevista 24. Montes Giraldo et al., 1997: $533)$.

Cuadro 6. El tipo de discurso y su relación con la interpretación referencial de uno (cuadro cruzado)

\begin{tabular}{|l|c|c|c|}
\hline \multicolumn{1}{|c|}{ Tipo de discurso } & Yo & Nosotros & General \\
\hline Narración & $\mathbf{8 7}$ & 45 & 7 \\
\hline Exposición y argumentación & $\mathbf{8 5}$ & $\mathbf{5 9}$ & 15 \\
\hline Conjeturas e hipótesis & $\mathbf{9 0}$ & $\mathbf{6 5}$ & 47 \\
\hline
\end{tabular}

De nuevo, dicha funcionalidad se reduce en las referencias más generales $(17 \%, 15 \%$ y $47 \%)$, es decir, en las situaciones basadas en conocimientos generalmente compartidos y no, necesariamente, en su propia experiencia u observación directa. Los pocos casos de este tipo consisten en conjeturas e hipótesis como en el ejemplo (28):

(28) - Y es que los que dirigen piensan es más en cómo sacar provecho, les gusta más lo de afuera, entonces, eso es...

-Y ahí se diría que es absolutamente político. O sea, $\underline{s i}$ uno tiene un buen puesto bien pago, para que ponerse a hacer bobadas (Entrevista 26, 2008).

Por último, y volviendo a los pesos probabilísticos de la clase de entrevista (cuadro 4), las entrevistas más informales y espontáneas favorecen uno (individual espontánea 0.62; diálogos entre conocidos 0.55 ), mientras que las entrevistas formales, menos interactivas y de trato distante, lo desfavo- 
recen (individual formal 0.40; casi monólogos 0.40). Es decir, es significativo tanto el nexo entre lo formal e informal como entre los conceptos de distancia y cercanía.

Cuadro 7. La clase de entrevista y la interpretación referencial de uno (cuadro cruzado)

\begin{tabular}{|l|c|c|c|}
\hline \multicolumn{1}{|c|}{ Clase de entrevista } & Yo & Nosotros & General \\
\hline Poca interacción & $\mathbf{8 4}$ & 32 & 12 \\
\hline Individual formal & $\mathbf{8 9}$ & 50 & 14 \\
\hline Individual espontánea & $\mathbf{8 6}$ & $\mathbf{5 9}$ & 27 \\
\hline Diálog. conocidos & $\mathbf{8 7}$ & $\mathbf{5 7}$ & 12 \\
\hline
\end{tabular}

\section{Conclusiones}

El análisis corrobora que los criterios semántico-pragmáticos de interpretación referencial, clase semántica del verbo y tipo de entrevista son determinantes del uso de uno en el español de Bogotá. La hipótesis inicial sobre un uso primordialmente subjetivo queda sustentada con la evidencia estadística acerca de la restricción de la característica de impersonalidad. Entre los indicios de este empleo de uno se encontraron los siguientes:

1. Se utiliza tanto con un sentido cercano a yo-hablante como en casos de pluralidad implícita con referencia a un nosotros. 
2. Se expresa de manera explícita $83 \%$ de los casos (la mayoría en función de sujeto), lo cual puede conducir a una interpretación más orientada al yo.

3. Se favorece principalmente con verbos estativos, emotivos y mentales (de opinión o de pensamiento) cuando refiere a la experiencia del hablante. Esta tendencia puede reflejar un doble propósito: el de enfocar la situación del yo-hablante y el de lograr la empatía de su interlocutor.

Comúnmente se ha señalado que uno funciona como un mecanismo de desfocalización, y su (sobre)uso se ha vinculado a las relaciones - de poder- entre los participantes, a su origen y a las formas de tratamiento. Sin embargo, el presente trabajo empírico apoya la idea de que en Bogotá predomina una fuerte conexión de uno con el hablante: habla de su experiencia con la posible intención de dirigir la atención hacia él mismo. De igual manera, le facilita ponerse en el lugar del otro y dejar huella de su perspectiva: uno como marca de la actitud del hablante frente a los eventos y situación de un grupo específico. Probablemente se trate de una tendencia a la subjetivización e intersubjetivización del discurso: de la actitud y el punto de vista del hablante, y del interés en crear un lazo de solidaridad o empatía en su interlocutor (Traugott, 2010: 32). En términos de la característica de evidencialidad mencionada en la introducción, los resultados apoyan la distinción entre la preferencia de uno para presentar información personal/privativa y se para la más universal (como en Fernández, 2008): uno se desfavorece en la expresión de referencias distanciadas y objetivas. 
Con todo, sería interesante poder seguir indagando hasta qué punto se está produciendo un reanálisis del significado semántico-pragmático de uno que pueda constituir un caso de subjetivización del discurso (Traugott, 2010): al parecer, tras la pérdida de la connotación de un uso de la clase popular, de procedencia rural y de un exceso inelegante, el bogotano lo está utilizando con las mismas funciones pronominales de $y o$.

Resultaría provechoso explorar qué dinámicas sociales se vinculan con este uso innovador, pues factores como la inmigración continua de colombianos de otras procedencias a la capital han ocasionado no solo un aumento de la población de Bogotá sino también cambios en los usos. Sería oportuno analizar la influencia del origen rural de los entrevistados o sus padres, pues la guerra civil colombiana, en curso desde los finales de la década del cuarenta (Montes Giraldo et al., 1998: 11), ha ocasionado la inmigración forzada del sector rural a la capital.

Ya que la variable que investigaba la relación entre los usos impersonales y los pronombres de tratamiento típicos de una región no fue estadísticamente significativa, es necesario seguir investigando otras variedades de español, considerando variables semántico-pragmáticas que precisen todavía más las causas del predominio de unas formas y/o el uso innovador de otras. Asimismo, sería interesante indagar qué ocurre en otras variedades cuyo uso ha sido asociado con hablantes de niveles socioeconómicos más bajos, como en el habla de Monterrey (Rodríguez Alfano, 2004).

Por último, parece indispensable continuar explorando factores como la transitividad de la acción, la posible in- 
fluencia de los verbos pronominales, la mención de acontecimientos negativos o positivos y si la construcción está enfocada en el participante o en el evento.

\section{Bibliografía}

Aikhenvald, Alexandra (2004), Evidentiality, Oxford, Oxford University Press.

Alfaraz, Gabriela (2015), "Variation of overt and null subject pronouns in the Spanish of Santo Domingo", en Ana Carvalho, Rafael Orozco y Naomi Lapidus (eds.), Subject Pronoun Expression in Spanish: A Cross-Dialectal Perspective, Washington, D.C., Georgetown University Press, pp. 3-16.

Arboleda, Rubén (2001), Oraciones impersonales con se: normas existentes, monografía de maestría, Bogotá, Instituto Caro y Cuervo.

Basa Vanrell, M. (2013), "Personal uno in Puerto Rican and Dominican Spanish", Studies in the Linguistic Sciences, pp. 1-20.

Briz, Antonio (2003), "La estrategia atenuadora en la conversación cotidiana española”, en Actas del Primer Coloquio del Programa EDICE, Estocolmo, Universidad de Estocolmo, pp. 17-46.

Cameron, Richard (1996), "A Community-based test of a linguistic hypothesis", Language in Society, 25, pp. 61111.

Carrasco, FÉlix (1988), "La indeterminación del sujeto en el español del siglo xvi”, en Actas del I Congreso Inter- 
nacional de Historia de la Lengua Española, Cáceres, Arco-Libros, pp. 319-328.

Casielles SuÁrez, Eugenia (1996), “Es la interpretación arbitraria realmente arbitraria?", Revista Española de Lingüística, 26, 2, pp. 359-377.

Company Company, Concepción (2010), "Reanálisis, ¿mecanismo necesario de la gramaticalización? Una propuesta desde la diacronía del objeto indirecto en español", RHLE, 5, pp. 35-36.

y Julia Pozas Loyo (2009), "Los indefinidos compuestos y los pronombres genéricos-impersonales omme y uno", en Concepción Company Company (dir.), Sintaxis histórica de la lengua española. Segunda parte: La frase nominal, México, Fondo de Cultura Económica y Universidad Nacional Autónoma de México, pp. 1073-1222.

Cuervo, Rufino José (1914), Apuntaciones críticas sobre el lenguaje bogotano, París, R. Roger y F. Chernoviz Editores.

DeMello, George (2000), “Tú impersonal en el habla culta”, Nueva Revista de Filología Hispánica, XLVIII, 2, pp. 359-372.

Dieck, Marianne (en prensa), “ ¿Y qué más hace uno, pues?: la expresión de la impersonalidad en el español de Medellín”, Revista Lingüística y Literatura, 69.

FERnández, Susana (2008), "Generalizaciones y evidencialidad en español", Revue Romane, 43, 2, pp. 217-234. Flores-Ferrán, Nidia (2008), "Are you refering to me? The variable use of uno and yo in the oral discourse", Journal of Pragmatics, 41, pp. 1810-1824. 
Gelabert-Desnoyer, Jaime (2008), "Not so impersonal: Intentionality in the use of pronoun uno in contemporary Spanish political discourse", Pragmatics, 18, 3, pp. 407-424.

González Vergara, Carlos y Evelin Hugo Rojas (2012), "Cuando te lo piden, uno no siempre sabe qué decir: uno y tú como estrategias evidenciales en el español de Chile", en Transformaciones culturales, debates de la teoría, la crítica y la lingüística en el Bicentenario, Actas del IV Congreso Internacional de Letras, Buenos Aires, Universidad de Buenos Aires, pp. 647-654.

Guantiva Acosta, Ricardo (2000), “Distribución del uso de las formas de tratamiento Pronominal tónica yo, mí, conmigo, e indefinida uno en el español hablado en Bogotá”, Forma y Función, 13, pp. 253-262.

Guirado, Kristel (2011), "La alternancia tú-uno impersonal en el habla de Caracas”, Lingüística, 26, pp. 2654.

Haßler, Gerda (2010), "Epistemic modality and evidentiality and their determination on a deictic basis", en Gabriele Diewald y Elena Smirnova (eds.), Empirical approaches to language typology: Realization of evidentiality in European languages, Berlín, Walter de Gruyter, pp. 223-248.

Haverkate, Henk (1985), "La desfocalización referencial en el español moderno", Hispanic Linguistics, 2, 1, pp. 1-21. (1987), "La cortesía como estrategia conversacional”, Diálogos Hispánicos de Ámsterdam, 6, pp. 27-63.

HEAD, BRIAN (1978), "Respect degrees in pronominal reference", en Joseph H. Greenberg, Charles A. Fergu- 
son y Edith A. Moravcsik (eds.), Universals of human languaje, Stanford, Stanford University Press, pp. 151211.

Hernanz, Ma. Lluïsa (1990), "En torno a los sujetos arbitrarios: la 2a persona del singular", en Violeta Demonte y Beatriz Garza Cuarón (eds.), Estudios de lingüística de España y México, México, Universidad Nacional Autónoma de México y El Colegio de México, pp. 151-178. Hollænder Jensen, Mikkel (2002), “La referencia en algunas expresiones impersonales: diferentes lecturas de uno y la segunda persona del singular", Romansk Forum, 16, pp. 127-138.

Hurtado, Luz Marcela (2005), "El uso de tú, usted y uno en el español de los colombianos y colomboamericanos", en Luis A. Ortiz y Manel Lacorte (eds.), Contactos y contextos linguisticos: el español en los Estados Unidos $y$ en contacto con otras lenguas, Madrid, Iberoamericana-Vervuert, pp. 187-200.

_ (2012), "La variable expresión de impersonalidad en el español de los colombianos de Bogotá y Miami”, en Richad File-Muriel y Rafael Orozco (eds.), Linguistic studies in Colombian varieties of Spanish, Madrid, Iberoamericana, pp. 141-167.

Kluge, Bettina (2010), "El uso de formas de tratamiento en las estrategias de generalización”, en Martin Hummel, Bettina Kluge y María Eugenia Vázquez (eds.), Formas y fórmulas de tratamiento en el mundo hispánico, México, El Colegio de México, pp. 1108-1136.

Martínez-Linares, Ma. Antonia (2009), "From hiding the speaker to persuasion: $\mathrm{Se}$-passive and se- imperso- 
nal constructions", Linguistic Insights: Studies in Language and Communication, 83, pp. 221-260.

Moltmann, Friederike (2006), "Generic one, arbitrary PRO, and the first person", Natural Language Semantics, 14, pp. 257-281.

Montes Giraldo, José Joaquín (1982), El español de Colombia: propuesta de clasificación dialectal, Bogotá, Instituto Caro y Cuervo.

- (1985), Estudios sobre el español de Colombia, Bogotá, Instituto Caro y Cuervo.

_ et al., (1997), El español hablado en Bogotá: relatos semilibres pertenecientes a tres estratos sociales, Bogotá, Instituto Caro y Cuervo.

— et al., (1998), El español hablado en Bogotá: análisis previo de su estratificación social, Bogotá, Instituto Caro y Cuervo.

Morales, Amparo (1995), “The loss of the Spanish impersonal particle se among bilinguals: A descriptive profile", en Carmen Silva-Corvalán (ed.), Spanish in the four continents, Washington D.C., Georgetown University Press, pp. 148-162.

MuñIz CACHón, CARMEN (1998), Impersonalidad y despersonalización: estudio contrastivo, Oviedo, Universidad de Oviedo.

Orozco, RAFAEL (2012), “The expression of nominal possession in the Spanish of Colombians in New York City", en Richad File-Muriel y Rafael Orozco (eds.), Linguistic studies in Colombian varieties of Spanish, Madrid, Iberoamericana, pp. 205-233. 
Orozco, Rafael y Richard File-Muriel (2012), “Colombian Spanish at the turn of the 21st century", en Richad File-Muriel y Rafael Orozco (eds.), Linguistic studies in Colombian varieties of Spanish, Madrid, Iberoamericana, pp. 11-20.

Otálora de Fernández, Hilda y Alonso González (1990), El habla de la ciudad de Bogotá: materiales para su estudio, Bogotá, Instituto Caro y Cuervo.

Posio, Pekкa (2011), "Spanish subject pronoun usage and verb semantics revisited", Journal of Pragmatics, 43, pp. 777-798.

Ricós Vidal, A. (2002). "Construcciones impersonales en el español medieval y clásico: estructuras con omne, se y uno", en Actas del V Congreso Internacional de Historia de la Lengua Española, Madrid, Gredos, pp. 945958.

Rodríguez Alfano, Lidia (2004), “Deixis personal: manifestación discursiva del sujeto y su ideología”, en ¿Qué opinas con verbos y pronombres? Análisis del discurso de dos grupos sociales de Monterrey, Monterrey, Universidad Autónoma de Nuevo León, pp. 151-214.

Siewierska, ANNA (2008a), "Impersonalization from a subject-centred vs. agent-centred pespective", Transactions of the Philological Society, 106, 2, pp. 115-137.

(2008b), "Ways of impersonalizing: pronominal vs. verbal strategies", en J. Lachian Mackenzie (ed.), Current trends in contrastive linguistics: Functional and cognitive perspectives, Ámsterdam, John Benjamins, pp. 3-16. 
Tagliamonte, Sali (2006), Analyzing sociolinguistic variation, Cambridge, Cambridge University Press.

Traugott, Elizabeth Closs (2010), “(Inter)subjectivity and (inter)subjectification: A reassessment", en Kristin Davidse, Lieven Vandelanotte y Hubert Cuyckens (eds.), Subjectification, intersubjectification and grammaticalization, Berlín, Mouton de Gruyter, pp. 29-71.

Vera Luján, Agustín (1990), Las construcciones pronominales pasivas e impersonales en español, Murcia, Universidad de Murcia. 
